\title{
Informational affordances: evidence of acquired perception-action sequences for information extraction
}

\author{
Irene Reppa • William C. Schmidt • Robert Ward
}

Published online: 28 February 2012

(C) Psychonomic Society, Inc. 2012

\begin{abstract}
Visual objects can automatically prime actions allowing efficient interaction with them. The present study examined whether object perception can automatically prime actions leading to efficient information extraction. Participants in Experiment 1 learned to rotate a cube in a specific way with the end goal of efficiently revealing object-identifying information. In Experiments 2 and 3, the end goal of obtaining object-identifying information was removed, but the stimulus-response associations were preserved. Only object views associated with actions learned in the context of obtaining identifying information caused response interference and benefits in a subsequent test phase where the object was irrelevant. These results demonstrate the existence of informational affordances: perception-action sequences acquired with the goal of information extraction that are automatically primed during later exposure to the object. Perceptual priming of actions for efficient information extraction is an important component of expert performance and its use of action systems to optimally deal with the world.
\end{abstract}

Keywords Acquired affordances · Action · Information · Perception

\footnotetext{
I. Reppa $(\bowtie)$

Wales Institute for Cognitive Neuroscience, Swansea University, Swansea, UK

e-mail: i.reppa@swansea.ac.uk

W. C. Schmidt

SR Research,

Ottawa, Canada

R. Ward

Wales Institute for Cognitive Neuroscience, Bangor University,

Bangor, UK

Recent work on affordances has revealed that object parts can automatically influence visuomotor behavior even when they and the object they belong to are irrelevant to the task at hand. For instance, a teacup may offer an affordance for grasping either by virtue of its typical function (e.g., Craighero, Fadiga, Rizzolatti, \& Umiltà, 1999; Ellis \& Tucker, 2000; Phillips \& Ward, 2002; Tucker \& Ellis, 1998; Vingerhoets, Vandamme, \& Vercammen, 2009) or simply by having an action-appropriate orientation (e.g., Symes, Ellis, \& Tucker, 2007). In the former sense, the teacup can be said to have a functional affordance - that is, a feature that facilitates efficient object-observer interaction (e.g., Gibson, 1979; Michaels, 1988).

Actions have goals and consequences. The influence of action consequences on action preparation and execution have been shown experimentally in a variety of tasks, including approach/avoidance (e.g., Bamford \& Ward, 2008; Seibt, Neumann, Nussinson, \& Strack, 2008; van Dantzig, Pecher, \& Zwaan, 2008), stimulus-response (S-R) compatibility (e.g., Grosjean \& Mordkoff, 2002; Guiard, 1983; Hommel, 1993), and imitation (e.g., Wohlschlägger, Gattis, $\&$ Bekkering, 2010). The effect of goal-related action consequences is illustrated by affordances, whereby multiple action possibilities are automatically activated during object perception. Current goals can determine whether and which actions will be activated on the basis of knowledge of their consequences (e.g., Michaels, 1988).

Apart from actions determined by typical object use, there are other actions that may be elicited by arbitrary object features. Consider, for example, the goal of making a rye bread loaf. Looking inside our cupboard, we may see a packet but be unable to determine whether the packet contains the rye flour we need or a different type of flour. To find the information we need about the packet's contents, we need to turn it in a certain way. Our experience with flour 
packets informs us that from the current position of the packet, there is an efficient and a less efficient way to get to the information. Extensive experience with this task for an individual who must carry it out often might result in the automatic activation of actions that will assist in optimizing subsequent informational input. Such perception-action sequences serve efficient information extraction and will be referred to as informational affordances.

Some striking examples implying that observers can acquire perception-action sequences aiding identification have come from work on perceptual expertise. Biederman and Shiffrar (1987) reported that expert chicken sexers differ from novices to the extent that the former know discriminating features to look for, where to look, and how to find them. Anecdotally, experts were taken aback when asked to identify chick sex from pictures, because they were accustomed to holding and manipulating the chicks to reveal discriminating information. This is a good example of an informational affordance: an acquired perception-action sequence that is contextually triggered to aid in the specific task of identifying the chick's sex.

The concept of informational affordances demands that the goal (and consequence) of obtaining information is a special type of goal and a critical factor in action. The observation that action goals and consequences can influence visuomotor behavior has been captured by ideomotor accounts of the perception-action link (e.g., Hommel, Müsseler, Aschersleben, \& Prinz, 2001; Jeannerod, 1988; Prinz, 1997). In contrast, sensorimotor accounts (e.g., Massaro, 1990) suggest that the stimulus-action association depends on the strength of reinforcement or repeated practice.

The present study employed a modified Simon task (Simon, 1969) sensitive to functional object affordances (see Phillips \& Ward, 2002). In three experiments, participants were trained to respond with an arbitrary left or right keypress to symmetrical shapes presented on the face of a cube. In Experiment 1 participants learned that the keypress would be correct if it facilitated fast identification, causing the cube to rotate along the shortest path to a discriminating cube face. In Experiments 2 and 3 participants were required to carry out exactly the same actions as in Experiment 1 . The critical difference was that following the keypress, in Experiment 2 there was no other goal apart from pressing the correct key (rotate the cube in the correct way), and in Experiment 3 the goal was to get to a certain cube face but not obtain information about it. Table 1 summarizes the three experiments.

In a test phase following learning in all three experiments, a speeded directional response to a target stimulus was required in the presence of the (now irrelevant) shapes seen during acquisition. Evidence for the transfer of automatic response activation was sought by testing for $\mathrm{S}-\mathrm{R}$ compatibility effects when the task-irrelevant cube face required the same lateralized response as during the learning phase (compatible trials) versus the opposite response (incompatible trials). Varying the stimulus onset asynchrony (SOA) between the prime and target during the test phase allowed assessment of the time course of any effects the prime might have.

Sensorimotor theories predicted a significant influence from responses associated with the irrelevant prime in all experiments, given that identical actions with identical feedback were required throughout. Ideomotor accounts, in contrast, predicted that automatic responses associated with the irrelevant primes would be elicited only if they were formed in the presence of an extrinsic goal (Experiments 1 and 3), but not in the absence of such a goal (Experiment 2). Finally, the concept of informational affordances was examined in terms of the difference between Experiments 1 and 3 .

\section{Experiment 1}

In Experiment 1, participants were trained to press one of two keys (left or right) with the goal of efficiently rotating a cube to reveal object identity information. In a subsequent test phase, the same cube faces encountered during learning appeared as irrelevant prime objects above a symbolic target requiring a left or right keypress response. The question was whether, during the test phase, the prime stimulus would automatically evoke a response that would allow it to be identified.

Method

\section{Participants}

Twenty naïve volunteers ( $M=26.0$ years, $S D=8.0$ years) participated in Experiment 1. ${ }^{1}$

\section{Stimuli and apparatus}

Two yellow surface-rendered cubes were created in Strata 3D Pro, containing a shape on each of their four adjacent faces (Fig. 1a). The two cubes were identical with respect to the shapes on three of their four faces (a cross, a rectangle, and a circle) but had a unique fourth face (a triangle on one cube, a star on the other). This is referred to as the

\footnotetext{
${ }^{1}$ To increase confidence in the results of the Experiment 1, a replication was undertaken, reproducing the same significant SOA by Compatibility interaction (particularly at later SOA). Although compatibility effects differed in magnitude from those reported here, significant between-experiment interactions held.
} 
Table 1 Summary of compatibility effects during the test phase of each experiment, as a function of learning goal (see text for more details)

\begin{tabular}{|c|c|c|c|c|}
\hline Experiment & Action Goal During Learning & Action Effect and Feedback & Secondary Task? & Compatibility Effect \\
\hline Experiment 1 & $\begin{array}{l}\text { Get to the discriminatory } \\
\text { face cube in the shortest } \\
\text { time... }\end{array}$ & $\begin{array}{l}\text { - Complete cube rotation } \\
\text { - Auditory positive or negative "beep" } \\
\text { - Written positive or negative statement }\end{array}$ & $\begin{array}{l}\ldots \text { then, report identity of } \\
\text { the shape on the discriminatory } \\
\text { cube face (star or triangle) }\end{array}$ & Yes \\
\hline Experiment 2 & $\begin{array}{l}\text { Rotate the cube in the } \\
\text { correct way (by pressing } \\
\text { the correct key). }\end{array}$ & $\begin{array}{l}\text { - Complete cube rotation } \\
\text { - Auditory positive or negative "beep" } \\
\text { - Written positive or negative statement }\end{array}$ & No secondary task & No \\
\hline Experiment 3 & $\begin{array}{l}\text { Get to the triangle (or the star) } \\
\text { in the shortest time... }\end{array}$ & $\begin{array}{l}\text { - Complete cube rotation } \\
\text { - Auditory positive or negative "beep" } \\
\text { - Written positive or negative statement }\end{array}$ & $\begin{array}{l}\text {. . then, report identity of the } \\
\text { shape on the initial cube } \\
\text { face (cross, circle, or square) }\end{array}$ & No \\
\hline
\end{tabular}

discriminatory face. From a viewing distance of $57 \mathrm{~cm}$, each cube subtended $6^{\circ} \times 6^{\circ}$ of visual angle, with $2.5^{\circ} \times 2.5^{\circ}$ shapes. Each cube was rendered in 12 different views by rotating it along its $y$-axis (Fig. 1b). During the learning phase, the cube was shown rotating from its initial to its final position at a rate of six images per second. During the test phase, centered target symbols were presented $0.5^{\circ}$ below the cube in Helvetica font $\left(0.7^{\circ} \times 2.0^{\circ}\right)$ and indicated the lateralized response to make. The response side indicated by the symbols was counterbalanced, with I-III indicating a left response and III-I a right response for half the participants and the reverse mapping for the other half of the participants.

\section{Procedure}

Learning phase Each trial began by showing one of the three nondiscriminatory sides of the cube (the cross-, rectangle-, or circle-containing side). Participants were given an unlimited amount of time to select either " $m$ " or " $z$," which would cause the cube to rotate counterclockwise or clockwise, respectively, around its $y$-axis (Fig. 1b). The task was to reach the discriminatory face by taking the shortest rotation path. If the shortest path between the initial object view and the discriminatory side was selected, positive auditory feedback accompanied the message "Good Work! That was the shortest path"; otherwise, negative auditory feedback accompanied the message "That wasn't the shortest path ... Keep trying!" On neutral-circle trials, the circle face appeared initially, and response feedback was always positive, since there was no shortest path to the discriminatory face. Following the cube's rotation and appropriate feedback, participants were asked to report the discriminatory face's shape, by pressing " $\mathrm{s}$ " if it was a star or " $\mathrm{t}$ " if it was a triangle. Responses to this task were recorded but were not part of the learning criterion (for this or the following experiments).
The learning criterion was 36 correct shortest path selections in a row for two consecutive learning blocks. Trial order within each block was randomly determined with selection from all combinations of discriminatory face and initial view.

Test phase In the test phase, a symbolic target indicating left or right (I-III or III-I) was presented 0,200 , or $800 \mathrm{~ms}$ after prime onset. Written instructions asked participants to ignore the prime and to respond quickly and accurately to the target by pressing the " $\mathrm{z}$ " or " $\mathrm{m}$ " key for the left or right target, respectively. One of five cube faces appeared above the target as an irrelevant prime (Fig. 2). The cross, rectangle, and circle had been associated with directional responses during learning, while the triangle and star had no directional association. Auditory feedback and a written message were supplied on the basis of the participant's response, and there was a 2,500-ms intertrial interval.

\section{Design}

Two within-subjects factors were manipulated in the test phase. The SOA factor had three levels: The prime would be presented at the same time as the target at 0 SOA or 200 or $800 \mathrm{~ms}$ after the target. The compatibility factor had four levels (compatible, incompatible, neutral circle, and neutral; see Fig. 2) relating the correct target response during the test phase to an earlier learned response to the cube's initial face. On compatible trials, the response associated with the prime and required by the target was the same (e.g., both left). On incompatible trials, the response was different.

Two different types of baseline trials were administered. On neutral trials, the prime was the discriminatory cube face containing either the star or the triangle, which were not explicitly linked with a directional (left or right) response during the learning phase. On neutral-circle trials, the prime 
Fig. 1 a Two cube stimuli had three identical adjacent faces and a unique discriminatory face (a triangle or a star). $\mathbf{b}$ Rotation path of the cube with a triangle on its discriminatory face, following a correct (left panel) and an incorrect (right panel) response
(A)

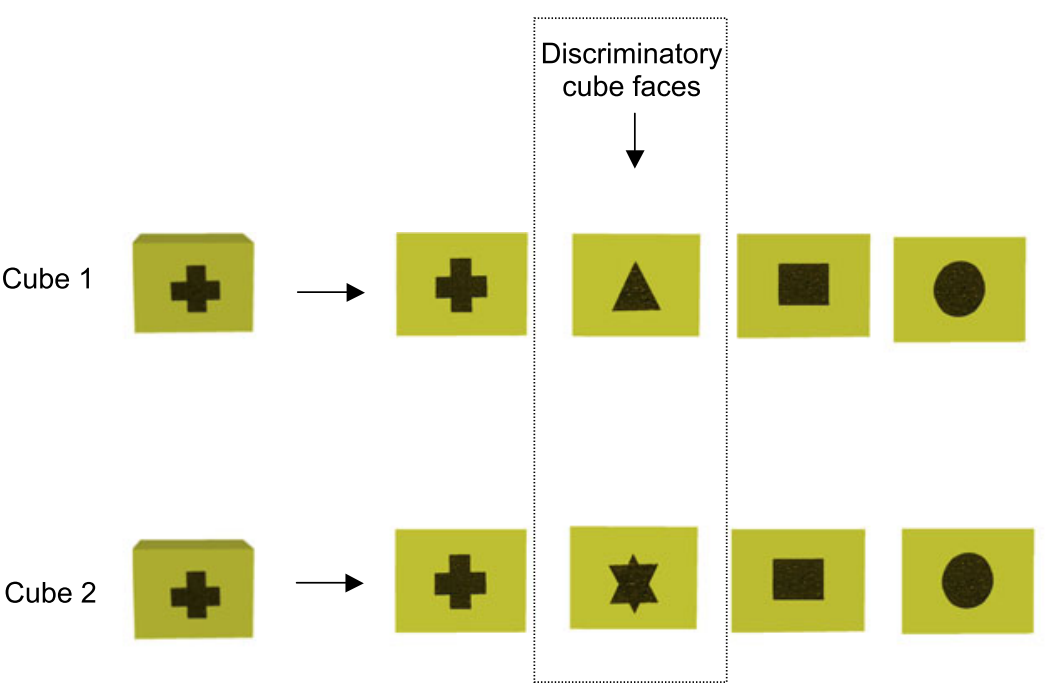

(B)
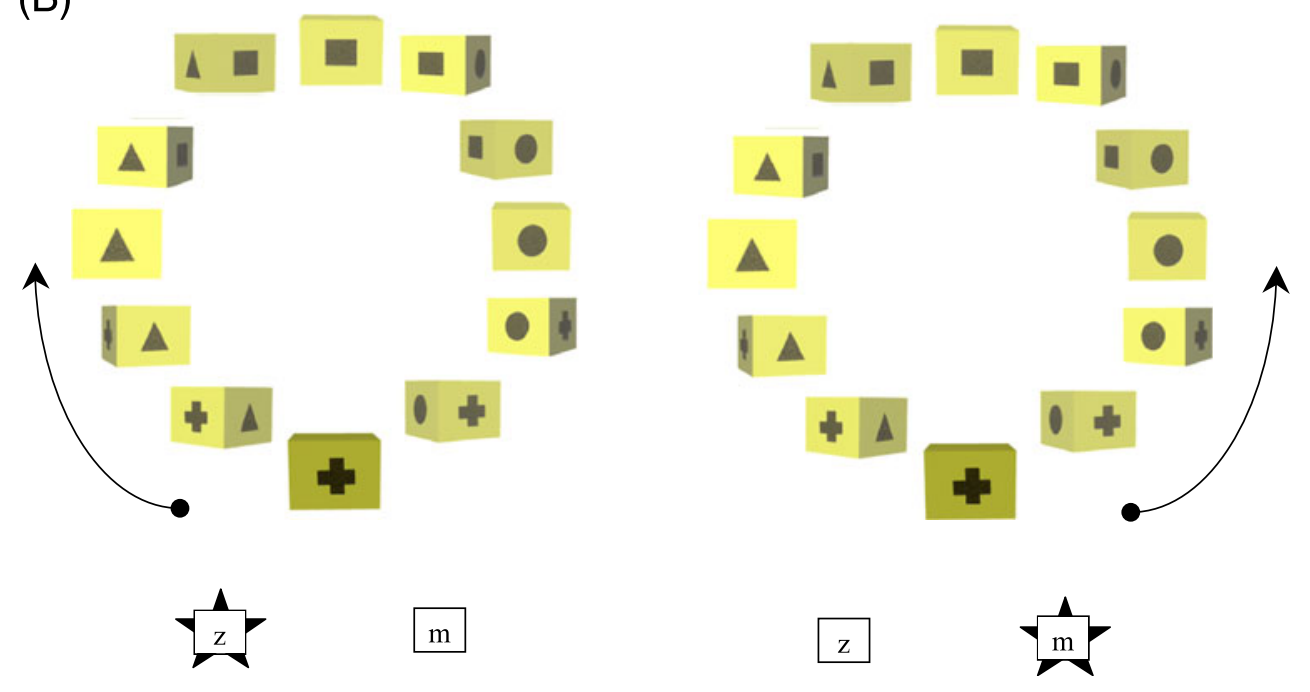

was the cube face containing the circle, which was equally

Results

associated with a left or a right directional response. There were 10 trials for each response (left and right), object face (star, triangle, rectangle, cross, and circle), and SOA (0, 200, $800 \mathrm{~ms}$ ), for a total of 300 trials. Trials were presented randomly in 10 blocks of 30 trials.

Learning data Mean trials to criterion are shown in Table 2.

Test data Trials with response times (RTs) greater than 2,500 $\mathrm{ms}$ and less than $200 \mathrm{~ms}$ were excluded as outliers

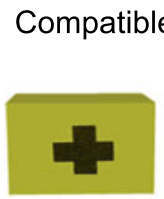

$|-|||$
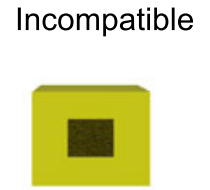

$|-|||$

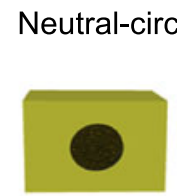

|-III

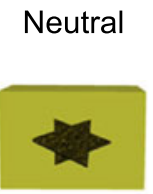

$|-| I \mid$
Neutral

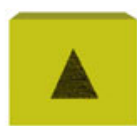

$|-| I \mid$
Fig. 2 Possible prime-target compatibility stimulus combinations during the test phase. In this example "I-III" means "left keypress." Compatible trials required the same keypress response to the stimulus as that required during learning. Incompatible trials required the nonacquired keypress response. On neutral-circle trials, the circle prime was shown, for which any directional responses were accepted during acquisition. Finally, on neutral trials, the prime image was either a star or a triangle, neither of which required any directional association during the learning phase 
Table 2 Mean number of trials and standard deviations (with $S D$ s) to reach learning criterion in each of the three experiments

\begin{tabular}{lll}
\hline Experiment & \multicolumn{2}{l}{ Mean Trials to Criterion $(S D)$} \\
\cline { 2 - 3 } & \multicolumn{1}{l}{ Block 1 } & \multicolumn{1}{l}{ Block 2 } \\
\hline Experiment 1 & $62.4(23.2)$ & $39.5(9.0)$ \\
Experiment 2 & $54.3(19.7)$ & $37.9(7.9)$ \\
Experiment 3 & $50.8(15.9)$ & $38.9(7.8)$ \\
\hline
\end{tabular}

$(0.15 \%)$. Errors accounted for $1.1 \%$ of all trials. Mean correct RTs were entered into two separate analyses using distinct neutral conditions. Cell means appear in Table 3. For neutralcircle trials, a 3 (SOA: 0, 200, or $800 \mathrm{~ms}$ ) $\times 3$ (compatibility: compatible, incompatible, or neutral-circle) repeated measures analysis of variance (ANOVA). The main effects of SOA, $\mathrm{F}(2,38)=10.78, p=.0001$, and compatibility, $\mathrm{F}(2,38)=$ $7.81, p=.001$, were significant, as was their interaction, $\mathrm{F}(4$, $76)=3.74, p=.008$. The same ANOVA with the neutral trials similarly showed significant main effects of $\operatorname{SOA}, F(2,38)=$ $13.41, p=.0001$, and compatibility, $F(2,38)=7.72, p=.002$, and a significant interaction, $F(4,76)=4.93, p=.001$.

The compatibility $\times$ SOA interactions in the two ANOVAs were investigated with Bonferroni corrected t-tests. As can be seen from Table 4, incompatible trials were significantly slower than compatible trials and both types of neutral trials at all SOAs. Compatible trials were faster than neutral-circle trials only at SOA 800. Mean compatibility effects (differences between compatible and incompatible conditions from the two types of neutral conditions) appear in Fig. 3.

\section{Discussion}

Experiment 1 suggests that an arbitrary stimulus can be trained to automatically evoke a response contributing to its accurate identification. Responding to a lateralized target was significantly influenced by the previously trained response to an irrelevant prime: When the trained and required responses were compatible (e.g., both left keypresses), target identification was speeded, as compared with when the responses were incompatible. Participants were faster to make a response corresponding to prior learned actions that, in another context, would have most efficiently revealed the prime object's identity. This was true even though the response had no effect on the prime object within the test phase and despite the fact that the prime object was irrelevant to the task. These findings suggest that idiosyncratic perceptual attributes, such as shape, can prime actions that relate to the goal of information acquisition. In other words, viewing an object can elicit not only functional, but also informational affordances.

An interesting suggestion in the data of Experiment 1 is that the magnitude of compatibility effects may have increased with the amount of preview time (SOA). Complicating conclusions on this front is always the issue about the appropriate neutral baseline to use (Jonides \& Mack, 1984). While neutral-circle trials did not have a specific response association trained during acquisition, it is still possible that participants may have surreptitiously learned responses to these stimuli, since any response was treated as correct. Neutral trials (star and triangle shapes) were present during learning but were not part of the learning criterion.

Analyses on the time course of compatibility effects in Experiment 1 showed a significant increase with exposure when assessed with neutral trials, $F(2,38)=7.12, p=.002$ (Fig. 3a), but no significant increase when assessed with learning-contaminated neutral-circle trials, $F(2,38)=2.28$, $p=.11$ (Fig. 3b). Computing compatibility on the basis of an average of the neutral conditions revealed significant increases with preexposure, $F(2,38)=4.88, p=.013$. An increase in compatibility effect magnitude as a function of exposure time would not be unwarranted, given that cognitive representations are presumably involved in the goal-related evaluation of the stimulus and may be helping to cause the increase in response benefit and interference magnitude over time.

\section{Experiment 2}

The hypothesis of informational affordances suggests that the compatibility effects seen in Experiment 1 reflect an automatic bias to make actions that aid object identification. However, there are other possibilities. Experiment 2 was conducted to ensure that the observed compatibility effects of Experiment 1 did not simply arise from S-R associations during learning. Ideomotor theory proposes that an action's perceptual consequences influence response selection, whereas sensorimotor accounts suggest that $\mathrm{S}-\mathrm{R}$ association, regardless of perceptual consequences, would be sufficient to influence response
Table 3 Experiment 1 mean RTs; (with $S D$ s in parentheses)

\begin{tabular}{|c|c|c|c|c|}
\hline \multirow[t]{2}{*}{ SOA } & \multicolumn{4}{|l|}{ Mean RT (ms) } \\
\hline & Compatible & Incompatible & Neutral Circle & Neutral \\
\hline $0 \mathrm{~ms}$ & $545.8(113.73)$ & $655.3(256.44)$ & $572.0(144.52)$ & $531.4(135.28)$ \\
\hline $200 \mathrm{~ms}$ & $496.8(104.11)$ & $629.33(261.57)$ & $521.0(125.91)$ & 480.5 (98.72) \\
\hline $800 \mathrm{~ms}$ & $461.9(100.75)$ & $659.8(312.75)$ & $523.1(134.67)$ & $451.4(101.65)$ \\
\hline
\end{tabular}


selection. To test the predictions of these accounts, in Experiment 2, participants were required to learn the correct directional keypress response on the basis of the initial face of the cube presented, but there was no instruction to determine the shortest path or identify the discriminating face of the cube.

Method

\section{Participants}

Twenty-one naïve volunteers $(\mathrm{M}=25.4$ years, $\mathrm{SD}=$ 9.1 years) participated in Experiment 2.

Stimuli, apparatus, and design

The stimuli, apparatus, and design were identical to those in Experiment 1.

\section{Procedure}

The procedure in was identical to that in Experiment 1, with the following exceptions. The instructions during the learning phase emphasized the importance of pressing the correct key (" $m$ " or " $z$ ") depending on the initial face, in order to rotate the cue in the correct way. There was no mention of a discriminatory face and no secondary task of identification. Therefore, although participants had to learn the correct S-R mapping, there was no indication as to why. The same learning criterion of 36 correct trials in a row in two consecutive blocks and the same auditory feedback were used as in Experiment 1, as well as a message saying either "Good work! That was the correct key," or "That wasn't the correct key... keep trying" for correct and incorrect responses, respectively. The test phase was identical to that in Experiment 1.

Results and discussion

Learning data

Mean trials to criterion are shown in Table 2.

\section{Test data}

Trials with RTs greater than $2,500 \mathrm{~ms}$ and less than $200 \mathrm{~ms}$ were excluded as outliers $(0.64 \%)$. Errors accounted for $1.74 \%$ of all trials. Cell means are shown in Table 5. A 3 (SOA: 0, 200, or $800 \mathrm{~ms}$ ) $\times 2$ (neutral type: neutral vs. neutral circle) repeated measures ANOVA on mean RT did not reveal a significant effect of neutral type or a significant interaction (both $F \mathrm{~s}<1$, n.s.); therefore, the two neutral baselines were averaged. A subsequent 3 (SOA: 0, 200, or $800 \mathrm{~ms}) \times 3$ (compatibility: compatible, incompatible, or neutral) repeated measures ANOVA revealed a significant 
Fig. 3 a Mean response times (RTs) in Experiment 1 and mean difference scores for compatible and incompatible conditions relative to the neutral-circle baseline, and $\mathbf{b}$ relative to the neutral baseline. Error bars indicate standard error of the mean
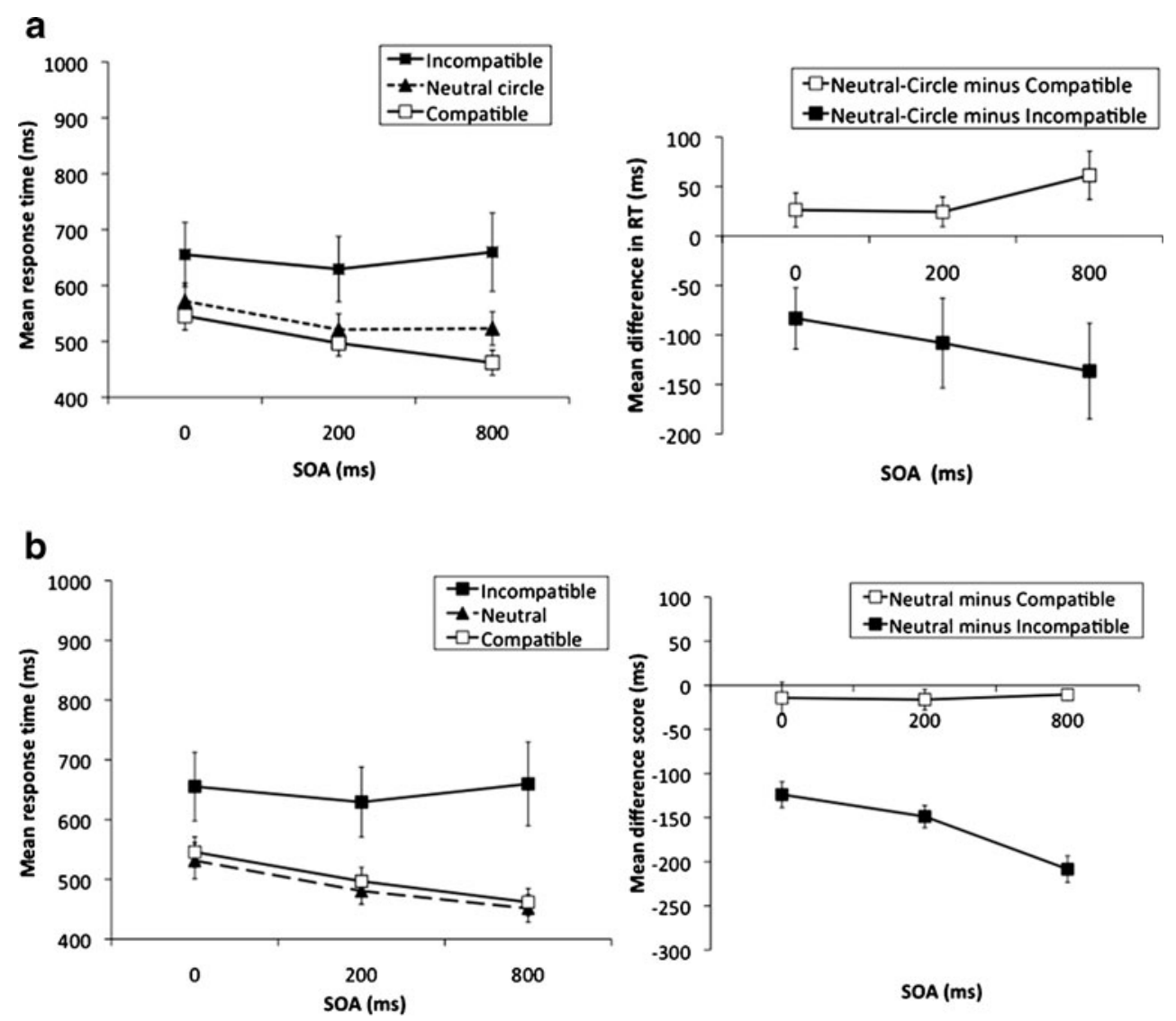

main effect of SOA, $\mathrm{F}(2,40)=20.23, p=.0001$, but the main effect of compatibility, $\mathrm{F}(2,40)<1, p>.05$, and the $\mathrm{SOA} \times$ compatibility interaction, $\mathrm{F}(4,80)<1, p>.05$, were not significant. Paired-sample $t$-tests contrasting levels of SOA revealed that the RT was significantly longer at SOA 0 than at $200 \mathrm{~ms}, t(20)=7.27, p=.0001$, and $800 \mathrm{~ms}, t(20)=$ $4.44, p=.0001$, while there was no difference between 200 and $800 \mathrm{~ms}, t(20)=0.37, p>.05$. Mean compatibility effects appear in Fig. 4.

Training participants to make $\mathrm{S}-\mathrm{R}$ associations in the absence of an end goal did not lead to any performance benefit when the associated cube face appeared as an irrelevant prime in the test phase. This finding suggests that compatibility effects in Experiment 1 resulted from the cube face eliciting the action most compatible with the efficient revealing of discriminatory information.

\section{Experiment 3}

The results so far support the notion of informational affordances: Responses acquired with the goal of obtaining identifying information can lead to significant compatibility effects in a later task, where they are no longer relevant. Nevertheless, it is possible that it is not the goal of identification that mediated later compatibility effects in Experiment 1, but the goal of "getting to" a cube face. That is, is it important that the target face contained information that discriminated object identity? The hypothesis of informational affordances suggests that this is crucial, that what participants are learning is the most effective response to achieve the goal of object identification. If the target face did not discriminate between possible object identities, we would no longer predict the automatic activation of responses to reveal the target face.

Method

\section{Participants}

Twenty naïve volunteers $(M=29.3$ years, $S D=11.33$ years $)$ participated in Experiment 3.

Table 5 Experiment 2 mean RTs (with $S D$ s in parentheses) per condition

\begin{tabular}{lllll}
\hline SOA & \multicolumn{4}{l}{ Mean RT (ms) } \\
\cline { 2 - 5 } & Compatible & Incompatible & Neutral Circle & Neutral \\
\hline $0 \mathrm{~ms}$ & $636.9(158.8)$ & $647.1(168.2)$ & $643.2(148.9)$ & $631.8(145.2)$ \\
$200 \mathrm{~ms}$ & $567.3(149.0)$ & $555.9(110.8)$ & $564.1(146.5)$ & $561.3(134.2)$ \\
$800 \mathrm{~ms}$ & $562.8(142.6)$ & $570.6(146.3)$ & $569.8(148.4)$ & $553.8(142.1)$ \\
\hline
\end{tabular}


Fig. 4 a Mean response times (RTs) in Experiment 2 and mean difference scores for compatible and incompatible conditions relative to the neutral-circle baseline, and $\mathbf{b}$ relative to the neutral baseline. Error bars indicate standard error of the mean
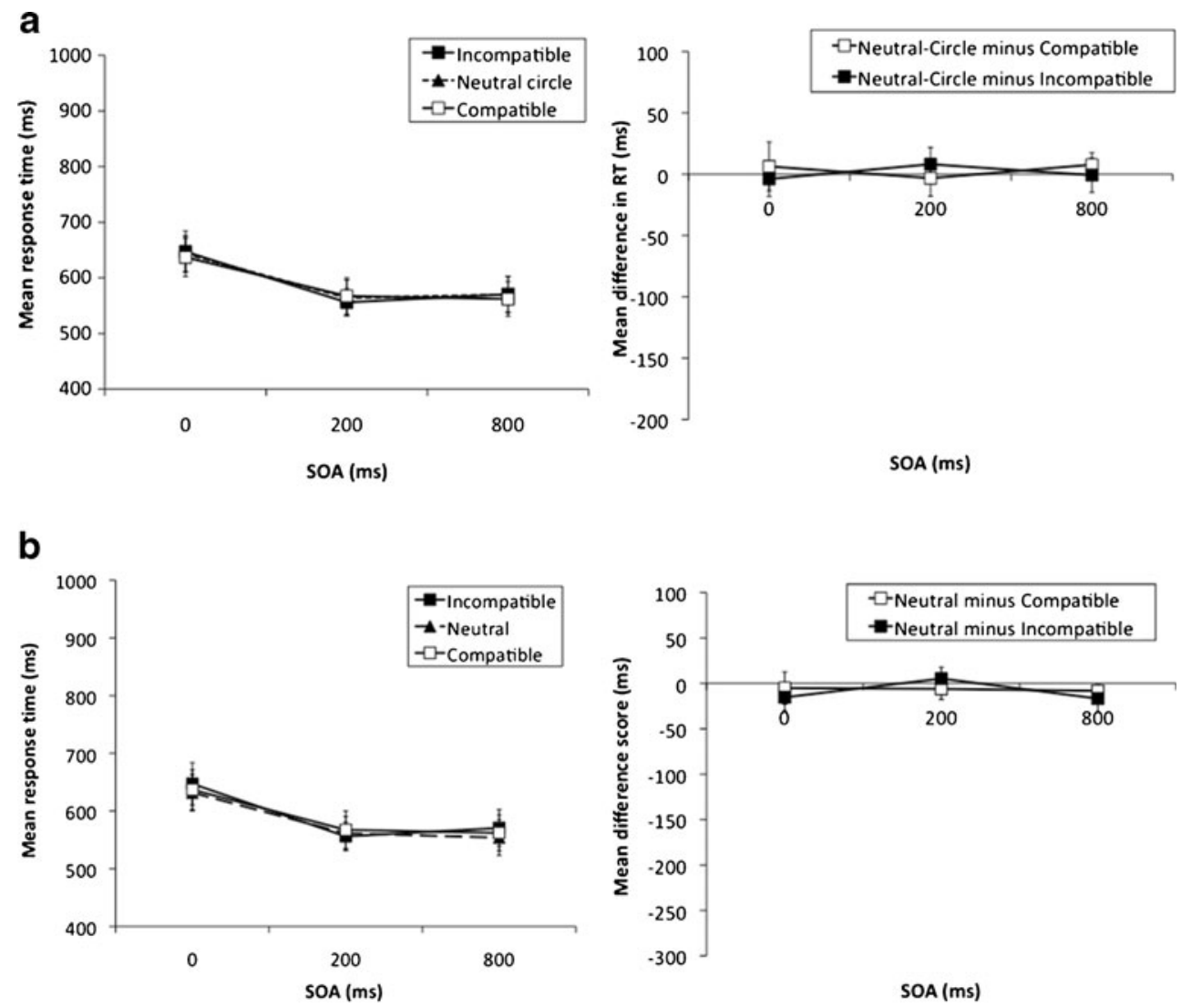

\section{Stimuli and apparatus}

The stimuli and apparatus were identical to those in Experiment 1.

\section{Design and procedure}

The learning procedure was similar to that in Experiment 1, with the following exceptions. Ten of the participants were presented with the triangle-containing cube only and were asked to rotate the cube using the shortest path in order to get to the face containing the triangle (get-to-the-triangle group). The other 10 participants were shown only the star-containing cubes and were asked to get to the face containing the star (get-to-thestar group). Following the keypress that rotated the cube, participants had the secondary task of reporting the identity of the initial face of the cube (press " $\mathrm{x}$ " for cross, "c" for circle, and "s" for square). The factor of group was analyzed as a between-subjects factor, but otherwise, the design was identical to that in Experiments 1 and 2. The learning criterion and feedback were identical to those in Experiment 1. The test procedure was the same as that in Experiments 1 and 2.
Results

Learning data

Mean trials to criterion are shown in Table 2.

\section{Test data}

Trials with RTs greater than 2,500 ms and less than $200 \mathrm{~ms}$ were excluded as outliers $(0.10 \%)$. Errors accounted for $1.5 \%$ of all trials. Cell means are shown in Table 6. The factor of group (getto-the-star or get-to-the-triangle) was not significant, nor was it involved in any interaction, so the data were collapsed across this factor. The two neutral baselines (neutral and neutral-circle) were combined into a single baseline, since the 3 (SOA: 0, 200, or $800 \mathrm{~ms}) \times 2$ (neutral type: neutral vs. neutral circle) repeated measures ANOVA did not reveal a significant effect of neutral type or a significant interaction (both $F_{\mathrm{S}}<1$, n.s.). A 3 (SOA: 0, 200 , or $800 \mathrm{~ms}$ ) $\times 3$ (compatibility: compatible, incompatible, or neutral) repeated measures ANOVA revealed a significant main effect of SOA, $\mathrm{F}(2,38)=53.14, p=.0001$, with RT significantly longer at SOA 0 than at $200 \mathrm{~ms}, t(19)=6.40, p=.0001$, and $800 \mathrm{~ms}, t(19)=9.68, p=.0001$, and RT longer at SOA 200 than at SOA $800 \mathrm{~ms}, t(19)=4.21, p=.0001$. Mean compatibility 
Table 6 Experiment 3 mean RTs (with $S D$ s in parentheses) per condition

\begin{tabular}{llcccc}
\hline \multirow{2}{*}{ Group } & \multicolumn{3}{l}{ Mean RT $(\mathrm{ms})$} & & \\
\cline { 2 - 6 } & $\mathrm{SOA}$ & Compatible & Incompatible & Neutral Circle & Neutral \\
\hline Star & $0 \mathrm{~ms}$ & $500.4(58.40)$ & $497.1(58.25)$ & $496.4(48.08)$ & $501.6(59.93)$ \\
& $200 \mathrm{~ms}$ & $465.1(52.90)$ & $453.7(47.26)$ & $460.5(51.33)$ & $459.0(53.03)$ \\
& $800 \mathrm{~ms}$ & $436.76(47.81)$ & $439.44(31.75)$ & $443.0(57.09)$ & $440.6(50.80)$ \\
Triangle & $0 \mathrm{~ms}$ & $543.0(83.65)$ & $555.3(104.88)$ & $554.9(95.05)$ & $543.2(88.04)$ \\
& $200 \mathrm{~ms}$ & $496.8(82.75)$ & $491.5(77.80)$ & $489.3(84.18)$ & $493.83(72.97)$ \\
Star and triangle combined & $0 \mathrm{~ms}$ & $521.7(73.53)$ & $526.2(87.80)$ & $525.6(79.22)$ & $522.4(76.34)$ \\
& $200 \mathrm{~ms}$ & $480.9(69.52)$ & $472.6(65.58)$ & $474.9(69.44)$ & $476.4(64.60$ \\
& $800 \mathrm{~ms}$ & $449.8(53.15)$ & $447.4(58.08)$ & $454.1(65.34)$ & $455.4(58.36)$
\end{tabular}

effects appear in Fig. 5. The main effect of compatibility, F(2, $38)<1, p>.05$ and the SOA $\times$ compatibility interaction, $\mathrm{F}(4$, $76)<1, p>.05$, were not significant.

Analysis between Experiments 1, 2, and 3

\section{Learning data}

A one-way ANOVA on the mean number of trials to criterion in the learning phase of the three experiments (Table 2) showed no significant main effect of experiment, $F(3,81)<$ $1, p>.05$.

\section{Test data}

A 3 (experiment: 1, 2, or 3) $\times 3$ (SOA: 0,200 , or $800 \mathrm{~ms}) \times 3$ (compatibility: compatible, incompatible, or neutral circle) mixed ANOVA on mean RT during the test phase revealed a significant main effect of SOA, $F(2,116)=60.54, p=.0001$, a significant main effect of compatibility, $F(2,116)=7.50$,
Fig. 5 a Mean response times

(RTs) in Experiment 3 and mean difference scores for compatible and incompatible conditions relative to the neutral-circle baseline, and $\mathbf{b}$ relative to the neutral baseline. Error bars indicate standard error of the mean
(A)
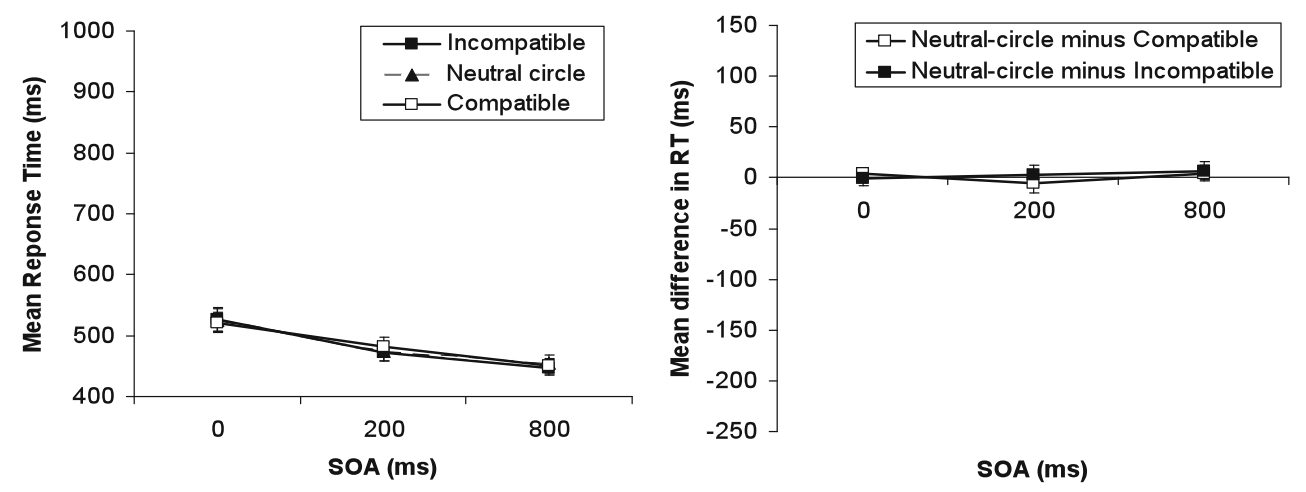

(B)

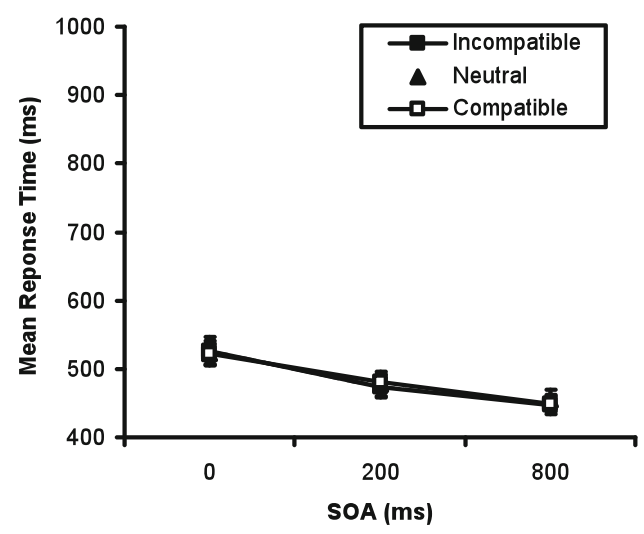

SOA (ms) 
$p=.0001$, and a significant main effect of experiment $F(2,58)=$ $4.03, p=.02$. The compatibility $\times$ experiment interaction was significant, $F(4,116)=7.57, p=.0001$. Apart from the marginally significant three-way interaction, $F(8,232)=1.87, p=.06$, there were no other significant interactions. The same pattern of results emerged when the neutral RTs replaced the neutral-circle RTs in the analysis. It is important to note that the compatibility $\times$ experiment interaction was driven solely by the difference between Experiment 1 and the other two experiments. These results confirm the informational affordances hypothesis: The goal of obtaining information (Experiment 1 ), as opposed to the goal of achieving the correct S-R mapping (Experiment 2) or the goal of getting to the information (Experiment 3), is unique in automatically activating actions in its service.

\section{Discussion}

Responses trained with the goal of getting to a cube face without obtaining information did not reveal any compatibility effects at a later test. This is a surprising finding, considering the evidence that end goals can influence performance (e.g., Hommel, 1993). The present results suggest that at least in the case of nonlateralized stimuli (and effects), responses are automatically elicited only after having been acquired with the goal of obtaining information.

\section{General discussion}

The present experiments demonstrate for the first time the concept of informational affordances: acquired perceptionaction sequences that aid in information extraction. In Experiment 1, task-irrelevant cube faces automatically elicited trained actions, which influenced responses to taskrelevant targets. Importantly, only response training in the service of the end goal of acquiring identifying information produced S-R compatibility effects. When the training did not require participants to engage with perceptual qualities of the stimulus in a goal-directed manner, or when the goal was to get to a cube face but not obtain any information from it, no interference or benefits occurred, despite no significant differences between experiments in the learning phase. Hence, the learned actions were automatically elicited by the stimulus only when such actions afforded efficient information extraction during acquisition. Even a liberal search in the data of Experiments 2 and 3 failed to show any influence of the presence of the primes on responding: Separate analyses on the short and long RTs following a median split of each participant's data revealed no sign of interference or benefits, even for the slowest of responses.

Importantly, symmetrical objects, naturally eliciting no spatial response, were trained to elicit compatibility effects when the associated action was acquired with the goal of obtaining information. To our knowledge, this is a unique finding in both the literature of affordances and the literature on the effect of action goals in action execution. Previous work on functional affordances confounded meaningful actions (i.e., picking up) with the side of space cued and acted upon (i.e., the handle could point to particular side of space) and afforded only a limited set of consequent actions (e.g., Phillips \& Ward, 2002). The present experiments separated meaning from spatial location, using symmetrical objects that would typically have no prior associated actions. The object view (a shape on a cube face) was associated with an arbitrary meaningful action via training.

Previous work on the effect of action goals has shown that goals such as approaching or avoiding a stimulus (e.g., Bamford \& Ward, 2008; Seibt et al., 2008; van Dantzig et al., 2008) or the goal of producing a lateralized stimulus (e.g., Guiard, 1983; Hommel, 1993) can produce S-R compatibility effects. The present findings show that $\mathrm{S}-\mathrm{R}$ compatibility effects can be produced even when the stimulus is nonlateralized (symmetrical cube) and the action effects relate only to the goal of efficient information extraction but have no other lateralized attribute (e.g., appearing close/ far, left/right).

The finding that trained object features elicited compatibility effects suggests that any object property could come to be associated with, and activate, very particular objectspecific skilled actions for the efficient extraction of perceptual information. Behavioral chains of such sequences might help explain the flexibility and optimization observed in expertise. Information in any form can be associated with actions providing increasingly efficient routes to further information extraction and subsequent actions, all contributing to optimal execution of a given task.

The present findings emphasize and provide empirical support for the exploratory nature of perception and its use of action systems to better perceive and identify the objects to act upon in the world. In terms of the sensorimotor approach to perception and action, perception dictates action. However, according to the ideomotor theory, we act not merely on what we see, but as a means to get to what we want to see (see also O'Regan \& Noe, 2001). Informational affordances empirically encapsulate this by showing that perception can dictate a special class of actions in service of perception itself and that associations driving these actions are highly flexible and very specific. It is clear from our experiments that having a goal of object identification changes the readiness of the visuomotor system to make certain actions. In particular, when in a task set related to object identification, actions that would facilitate identification are activated. The actions activated by an object must be specified at the level of goals (e.g., rotate the cube along 
the best path), not motor commands (e.g., press the left button). Just as perception for action emphasizes that sensory systems have evolved to enable action, the kind of action for perception illustrated in our results emphasizes that identifying the objects in our environment is an active, not a passive process.

Author note We gratefully acknowledge the help of the Wales Institute for Cognitive Neuroscience (WICN) for funding this project, as well as Michael Scott-Evans and Kate Williams for their help with data collection. Please address any correspondence regarding this article to Irene Reppa (i.reppa@swansea.ac.uk).

\section{References}

Bamford, S., \& Ward, R. (2008). Predispositions to approach and avoid are contextually sensitive and goal dependent. Emotion, 8, 174-183.

Biederman, I., \& Shiffrar, M. M. (1987). Sexing day-old chicks: A case study and expert systems analysis of a difficult perceptuallearning task. Journal of Experimental Psychology: Learning, Memory, and Cognition, 13, 640-645.

Craighero, L., Fadiga, L., Rizzolatti, G., \& Umiltà, C. (1999). Movement for perception: A motor-visual attentional effect. Journal of Experimental Psychology: Human Perception and Performance, $25,498-511$.

Ellis, R., \& Tucker, M. (2000). Micro-affordances: The potentiation of components of action by seen objects. British Journal of Psychology, 91, 451-471.

Gibson, J. J. (1979). The ecological approach to visual perception. Boston: Houghton Mifflin.

Grosjean, M., \& Mordkoff, J. T. (2002). Post-response stimulation and the Simon effect: Further evidence of action-effect integration. Visual Cognition, 9, 528-529.

Guiard, Y. (1983). The lateral coding of rotations: A study of the Simon effect with wheel- rotation responses. Journal of Motor Behavior, 15, 331-342.

Hommel, B. (1993). Inverting the Simon effect by intention: Determinants of direction and extent of effects of irrelevant spatial information. Psychological Research/ Psychologische Forschung, 55, 270-279.
Hommel, B., Müsseler, J., Aschersleben, G., \& Prinz, W. (2001). The theory of event coding (TEC). Behavioral and Brain Sciences, 24, 849-937.

Jeannerod, M. (1988). The neural and behavioural organisation of goal-directed movements. Oxford Psychology Press, 15.

Jonides, J., \& Mack, R. (1984). On the cost and benefit of the cost and benefit. Psychological Bulletin, 96, 29-44.

Massaro, D. W. (1990). An information-processing analysis of perception and action. In O. Neumann \& W. Prinz (Eds.), Relationships between perception and action: Current approaches (pp. 133166). Berlin: Springer.

Michaels, C. F. (1988). S-R compatibility between response position and destination of apparent motion: Evidence of the detection of affordance. Journal of Experimental Psychology: Human Perception and Performance, 14, 231-240.

O'Regan, J. K., \& Noe, A. (2001). A sensorimotor account of vision and visual consciousness. Behavioral and Brain Sciences, 24, 939-1031.

Phillips, J. C., \& Ward, R. (2002). S-R correspondence effects of irrelevant visual affordance: Time course and specificity of response activation. Visual Cognition, 9, 540-558.

Prinz, W. (1997). Perception and action planning. European Journal of Cognitive Psychology, 9, 129-154.

Seibt, B., Neumann, R., Nussinson, R., \& Strack, F. (2008). Movement direction or change in distance? Self- and object-related approach-avoidance motions. Journal of Experimental Social Psychology, 44, 713-720.

Simon, J. R. (1969). Reactions toward the source of stimulation. Journal of Experimental Psychology, 81, 174-176.

Symes, E., Ellis, R., \& Tucker, M. (2007). Visual object affordances: Object orientation. Acta Psychologica, 124, 238-255.

Tucker, M., \& Ellis, R. (1998). On the relations between seen objects and components of potential actions. Journal of Experimental Psychology: Human Perception and Performance, 24, 830-846.

van Dantzig, S., Pecher, D., \& Zwaan, R. A. (2008). Approach and avoidance as action effects. Quarterly Journal of Experimental Psychology, 61, 1298-1306.

Vingerhoets, G., Vandamme, K., \& Vercammen, A. (2009). Conceptual and physical object qualities contribute differently to motor affordances. Brain and Cognition, 69, 481-489.

Wohlschlägger, A., Gattis, M., \& Bekkering, H. (2010). Action generation and action perception in imitation: An instance of the ideomotor principle. Philosophical Transactions of the Royal Society of London B, 358, 501-515. 